\title{
Philosophiques
}

\section{La philosophie analytique de la religion : contribution canadienne 1970-1975}

\section{Benoît Garceau}

Volume 2, numéro 2, octobre 1975

URI : https://id.erudit.org/iderudit/203035ar

DOI : https://doi.org/10.7202/203035ar

Aller au sommaire du numéro

Éditeur(s)

Société de philosophie du Québec

ISSN

0316-2923 (imprimé)

1492-1391 (numérique)

Découvrir la revue

Citer cet article

Garceau, B. (1975). La philosophie analytique de la religion : contribution canadienne 1970-1975. Philosophiques, 2(2), 301-339.

https://doi.org/10.7202/203035ar d'utilisation que vous pouvez consulter en ligne.

https://apropos.erudit.org/fr/usagers/politique-dutilisation/ 


\title{
BULLETIN
}

\section{LA PHILOSOPHIE ANALYTIQUE DE LA RELIGION : CONTRIBUTION CANADIENNE 1970-1975}

\author{
par Benoît Garceau
}

L'un des domaines où la production a été le plus abondant chez les philosophes canadiens d'expression anglaise, au cours des cinq dernières années, est celui de la philosophie de la religion. Il m'a semblé pouvoir rendre service au lecteur de Pbilosopbiques en tentant de lui présenter un bilan critique de cette production. Je limiterai mon analyse aux ouvrages des philosophes qui exercent présentement leur activité dans une université canadienne et qui, de façon plus ou moins directe, s'inscrivent dans la tradition analytique.

L'examen de ces ouvrages appelle quelques remarques préliminaires. Depuis un quart de siècle, la philosophie analytique de la religion est aux prises avec deux problèmes principaux: celui du rapport entre la philosophie et la religion et celui du statut logique des énoncés de foi. Ces problèmes ne sont pas nouveaux, mais dans la philosophie analytique une façon nouvelle de les poser et de les discuter a pris forme. Pour la comprendre, il me paraît indispensable de rappeler deux événements qui l'ont profondément déterminée: la révolution opérée à l'intérieur de la philosophie analytique par l'œuvre du second Wittgenstein et le débat introduit par A. Flew sur la falsifiabilité des énoncés de foi. ${ }^{1}$

On reconnaît généralement à Wittgenstein d'avoir inspiré les deux périodes de la philosophie analytique : l'empirisme (ou positivisme) logique, par son Tractatus et l'analyse conceptuelle,

1. On trouvera une bonne introduction à ces problèmes dans N. O. SCHEDLER, Philosopby of Religion. Contemporary Perspectives, New York, Macmillan, 1974, pp. 219-250. 
par ses Pbilosophical Investigations. Remarquons tout de suite que la coupure entre les deux périodes n'a pas été totale; une même conception de la philosophie, comme clarification du discours plutôt qu'ensemble de thèses rivalisant avec celles de la science, comme activité plutôt que théorie, comme analyse du sens plutôt que synthèse métaphysique, en a assuré la continuité. Ce qui a changé, à l'intérieur de la philosophie analytique, c'est la théorie du langage et le critère du sens à partir desquels on a pratiqué l'analyse du discours.

Le positivisme logique se servait d'une théorie atomistique du langage, selon laquelle celui-ci est fait de propositions décomposables en propositions élémentaires dont les termes ne font que rapporter des données de l'expérience sensible. S'appuyant sur cette théorie réductionniste, il se donnait un critère fort simple permettant de filtrer les propositions dotées de sens. C'est le célèbre critère de vérifiabilité, dont on s'est servi, surtout chez les disciples d'A. J. Ayer, comme d'un bulldozer pour déraciner toute irruption de la métaphysique dans le langage. Une proposition n'a de sens, selon ce principe, que si elle est empiriquement vérifiable, à moins qu'elle ne soit analytique. Hors les propositions analytiques, qui ne disent rien du monde et ne font que préciser l'usage à faire de certains termes, et les propositions empiriques, que l'on peut vérifier en se rapportant aux faits d'expérience, il n'y a que des pseudo-propositions, «insensées», «émotives», «non-sens ». C'est notamment le cas des énoncés métaphysiques et religieux. Le positivisme logique ne les accusait pas d'erreur; il les excluait simplement de l'ordre des propositions sensées, seules susceptibles d'être vraies.

Le second Wittgenstein ${ }^{2}$ a introduit une autre théorie du

2. Puisque le présent bulletin a pour objet la contribution canadienne à la philosophie analytique de la religion, je me permets de mentionner ici l'excellente étude de Jacques POULAIN, de l'Université de Montréal, Le mysticisme du Tractatus Logico-Pbilosopbicus et la situation paradoxale des propositions religieuses, dans $L a$ recherche en pbilosophie et en théologie, ouvrage collectif édité par D. Dubarle, Paris, Cerf, 1970, pp. 75-155, de même que son ouvrage important Logique et Religion. L'atomisme logique de Wittgenstein et la possibilité des propositions religieuses; Paris, La Haye, Mouton, 1973. $\mathrm{La}$ philosophie analytique de la religion a également fait l'objet de la thèse de Pierre Lucier, Empirisme logique et langage religieux. Trois approches anglo-saxonnes contemporaines: R.B. Braithwaite, R.M. Hare, I.T. Ramsey, Lille, 1975, 2 vol. 828 p. en voie d'édition chez Desclée-Bellarmin. 
langage, appelée contextuelle, qui a paru à plusieurs analystes réhabiliter le langage religieux et rendre possible, pour la première fois, une véritable philosophie de la religion. Aux yeux de ces analystes de la deuxième génération, l'erreur du positivisme logique a été de s'intéresser à la seule fonction déclarative du langage, celle qui caractérise le discours scientifique, et de prescrire à partir de ce modèle les règles auxquelles toute proposition doit se plier pour avoir un sens. Il a oublié que le langage a d'autres fonctions, outre celle de constater un fait. Le commandement, la prière, le souhait, la promesse, sont des formes linguistiques et on ne peut nier qu'elles soient intelligibles. Ce qui fait le sens d'un mot ou d'un énoncé, c'est donc l'emploi qu'on en fait et chercher à comprendre ce sens c'est s'obliger à dévoiler les règles qui régissent leur emploi dans tel ou tel contexte de l'activité humaine.

À partir de cette théorie du langage, plusieurs analystes ont cru que la philosophie de la relig:on était enfin devenue possible, à condition de la limiter à la pure description des façons diverses de se servir du langage dans le contexte religieux, et à condition également que celui qui entreprend cette description connaisse par expérience le jeu du langage de la religion. L'adoption de ce point de vue n'était pas sans soulever un problème grave : celui du rapport entre la philosophie et la foi. La pure description du langage religieux n'oblige-t-elle pas le philosophe à renoncer à sa neutralité ? S'il doit en effet s'interdire de juger à partir de critères universels de la validité des expressions et des énoncés de la foi, pour limiter son travail à clarifier le sens de ces expressions et de ces énoncés dans la vie du croyant, n'adopte-t-il pas une position irrémédiablement fidéiste? Ne se condamne-t-il pas à entretenir une philosophie marquée par une sorte de provincialisme rationnel, qu'il pourra bien continuer d'appeler une philosophie de la religion, qui ne sera rien d'autre cependant qu'une philosophie religieuse ou une théologie, partie de la foi et visant à en élucider le sens?

Pour assurer la neutralité et l'autonomie de la raison dans l'analyse de la religion, la stratégie de plusieurs analystes a consisté à montrer que l'abandon du positivisme logique n'impliquait pas le rejet du principe de vérifiabilité. Tout en reconnaissant qu'il ne vaut plus comme règle universelle de sens, du 
fait que le langage sert à autre chose qu'à constater des faits, ils ont soutenu qu'il demeure le principe auquel on ne peut se soustraire pour juger si une phrase a une valeur cognitive, c'est-à-dire si elle traduit une prétention au savoir et non un pur état affectif, et si elle peut dès lors être légitimement considérée comme candidate à la vérité ou à l'erreur. ${ }^{3}$

Le problème du statut logique des énoncés de foi, deuxième problème capital dans la philosophie analytique de la religion, vient en grande partie de l'adoption de cette stratégie. Il reçut sa première formulation lors d'une discussion entre chrétiens et agnostiques, à l'Oxford University Socratic Club, en $1952 .{ }^{4}$ Dans un court exposé, A. Flew mettait les théologiens au défi de montrer qu'un énoncé sur Dieu puisse réellement affirmer quelque chose. Il n'y réussira pas, prescrivait Flew, tant qu'il n'aura pas défini de quelle manière cet énoncé pourrait être falsifié. Car un énoncé qui ne nie rien, écrivait-il, n'affirme rien, et si l'on ne peut rien concevoir qui réfute cet énoncé, il faut reconnaître qu'il ne constitue pas, malgré ses apparences, une véritable proposition. Bref, un énoncé théologique, s'il ne passe pas le test de la falsifiabilité, ne peut pas être tenu pour une proposition ayant une valeur cognitive, et par conséquent ne peut être admis comme candidat à la vérité. ${ }^{5}$

3. Voir BLACKSTONE, W. T. The Prablem of Religious Knowledge. The Impact of Pbilosophical Analysis and the Question of Religious Knowledge, Prentice-Hall, Englewood-Cliffs, N.J., 1963, surtout c. 8.

4. D'abord publiée dans The Socratic, V, Oxford, Basil Blackwell, 1952, cette discussion fut ensuite éditée avec d'autres études dans A. FLEW et A. MACINTYRE, New Essays in Pbilosopbical Tbeology, London, SCM Press, 1955, sous le titre "Theology and Falsification 》, pp. 96-130.

5. Essays in Theological Pbilosopby, p. 98. À noter que le critère de falsifiabilité fut d'abord proposé en 1934 par Karl POPPER, Logik der Forscbung, comme critère de démarcation entre ce qui est scientifique et ce qui ne l'est pas. Popper s'est toujours élevé contre l'emploi par les analystes du critère de falsifiabilité comme critère de sens. Voir sa réponse récente à ses critiques, dans The Pbilosopby of Karl Popper, ed. by P. A. Schilpp, The Library of Living Philosophers, Vol. XIV, Open Court Publishing Co., La Salle, Ill., 1974, Book II, pp. 965 ss. Chez Flew, le critère de falsifiabilité n'est ni un critère de sens, ni le critère de démarcation entre la science et la pseudoscience, mais le critère des énoncés ayant une valeur cognitive. Ce qui, à mon avis, constitue une manœuvre habile pour retenir l'essentiel de l'empirisme logique dans la pratique de l'analyse conceptuelle. 
Le défi de Flew a suscité chez les analystes une activité fébrile, un véritable «theological flu» impossible à enrayer. ${ }^{6}$ Pour défendre la validité des énoncés de foi, les théologiens et les philosophes ont eu recruurs à trois stratégies différentes, qu'il est bon de se rappeler en lisant les travaux de nos analystes canadiens, qui se définissent à peu près tous par rapport à ce défi. Une première stratégie consistait à refuser le critère de falsifiabilité, en disant qu'on ne peut l'appliquer aux énoncés théologiques qu'en violant leur intégtité, en les forçant à être jugés d'après un critère qui ne vaut que pour les énoncés de sciences empiriques. Une deuxième stratégie fut celle des théologiens de gauche (R. M. Hare, J.J.C. Smart, R. F. Holland, T. McPherson, R. B. Braithwaite, R. Hepburn, A. MacIntyre), ceux que Blackstone qualifie de positivisles cbrétiens, ${ }^{7}$ qui admettent le critère de falsifiabilité et reconnaissent que les énoncés théologiques ne répondent pas à ses exigences et doivent être tenus pour l'expression non pas d'un savoir, mais d'une attitude, d'un engagement, d'un point de vue. La troisième stratégie, celle des théologiens de droite (J. M. Crombie, B. Mitchell, J. Hick), a consisté à admettre le critère de falsifiabilité et à soutenir que les énoncés théologiques sont en principe falsifiables et qu'il faut par conséquent leur reconnaitre une valeur cognitive et un contenu empirique.

Les travaux que nous allons analyser développent l'une ou l'autre de ces positions. Ceux de K. Nielsen reprennent et précisent la stratégie d'A. Flew: celle du pratiquant de l'analyse conceptuelle qui ne renonce pas tout à fait à l'empirisme logique et à son critère de vérifiabilité et qui à sa lumière s'applique à montrer l'inintelligibilité du langage religieux. Les travaux de T. Penelhum exposent ensuite un point de vue assez voisin de celui des théologiens de la droite, celui de J. Hick notamment, avec un effort tout à fait nouveau cependant pour préserver jusqu'au bout la neutralité de la raison philosophique dans l'analyse de la religion. Disons tout de suite que dans la production canadienne analytique de la religion, on ne trouve aucun représentant de la théologie de gauche, probablement parce que la fragilité

6. Voir N. O. SCHEDLER, loc. cit., p. 230.

7. Voir W. T. BLACKSTONE, loc. cit., p. 66. 
de cette position en a bientôt fait une thèse indéfendable. L'ouvrage d'A. McKinnon développe de façon originale le point de vue du philosophe qui refuse de soumettre le langage de la foi à la critériologie falsificationiste et cherche à comprendre le sens de ce langage à partir de son propre contexte. ${ }^{8}$ Nous mentionnons enfin l'étude de J. King-Farlow qui, bien qu'elle ne constitue pas une contribution à la philosophie proprement analytique de la religion, indique cependant une nouvelle attitude, suggérée par le fallibilisme popperien, devant le débat sur le statut logique des énoncés de foi.

\section{1. $K$. Nielsen et la défense de l'athéisme}

Les nombreux travaux de $\mathrm{K}$. Nielsen en philosophie de la religion ${ }^{9}$ sont l'œuvre d'un philosophe engagé. Démontrer par les ressources de l'analyse conceptuelle l'absurdité de la religion,

8. Il m'a fallu à regret omettre la contribution de D. EVANS à la philosophie analytique de la religion. Son ouvrage The Logic of Self-Involvement. A Pbilosopbical Study of Everyday Language with Reference to the Cbristian use of Language about God as Creator, London, SCM Press, 1963, 293 pp., déborde les cadres de ce bulletin. Jean LADRIÈRE en a donné un excellent résumé dans «Langage auto-inplicatif et langage biblique selon Evans», dans Tijdschrift von Filosofie (Leuven), 28 (1966), reproduit dans L'Articulation du Sens. Discours scientifique et parole de la foi, Paris, Aubier - Cerf Delachaux et Nestié -- Desclée de B., 1970, pp. 91-139. Par ailleurs, l'ouvrage d'Evans, Religion and Morality, annoncé par la collection Pbilosopby of Religion Series, éditée par J. Hick chez Macmillan de Londres, n'était pas sorti des presses au moment de la rédaction de ce bulletin. L'œuvre d'Evans consiste à explorer dans l'esprit de la théorie contextuelle du sens et surtout de la théorie du langage performatif d'Austin les différents aspects du langage religieux. Parmi ses contributions récentes: «Faith and Belief », in Religious Studies, 10 (1974), 1-19, 199-212; "Preller's Analogy of Being 》, in New Scholasticism, 45 (1971), 1-37; A Reply to Flew's «The Presumption of Atbeism》, in Can. J. of Pbil., 2 (1972), 47.50; «Differences between Scientific and Religious Assertions », in N. O. Schedler, Pbilosopby of Religion. Contemporary Perspectives, N.Y., Macmilian, 1974, 305-327.

9. Outre les trois volumes analysés ici, Contemporary Critiques of Religion, London, Macmillan, «Philosophy of Religion Series 》, ed. by J. Hick, 1971, 163 p.; Etbics witbout God, London, Pemberton Books, 1972, 103 p.; Scepticism, «New Studies in the Philosophy of Religion», ed. by W. D. Hudson, London, Macmillan, 1973, NIELSEN a publié une quarantaine d'articles en philosophie de la religion. On trouvera la mention des plus importants dans Contemporary Critiques, pp. 157-158. Notons son premier ouvrage, Reason and Practice. A Modern Introduction to Pbilosopby, N:Y., Harper and Row, 1971,534 p. La troisième partie est consacrée à l'analyse du discours de la religion, pp. 135-227. 
expliquer ensuite le fait de la persistance chez l'homme de la croyance en Dieu, travailler enfin à l'affranchir de son besoin de religion, voilà le programme dans lequel s'inscrivent ses critiques répétées du langage de la foi. ${ }^{10}$ Est-ce à dire que la philosophie analytique se transforme chez lui en outil logique au service d'une idéologie? Certains aveux de Nielsen peuvent donner à le penser, par exemple lorsqu'il confesse que l'athéisme a été chez lui une conviction très ancienne qui a survécu aux changements d'orientation de sa philosophie. ${ }^{11}$ C'est cependant à sa thèse souvent formulée sur le rapport entre l'analyse conceptuelle et l'idéologie qu'il faut se reporter pour voir comment il espère assurer à la philosophie le pouvoir de critiquer la religion sans s'asservir à la justification rationnelle d'une autre idéologie. ${ }^{12}$ Pour Nielsen, en effet, l'analyse conceptuelle est idéologiquement neutre et elle ne l'est pas. Elle est idéologiquement neutre, en ce sens qu'elle trouve en elle seule la norme de sa réussite, indépendamment de tout présupposé idéologique, de toute conception métaphysique préalable du monde. Elle n'est cependant pas idéologiquement neutre si l'on entend par là qu'elle doit limiter sa tâche à la pure description des jeux du langage, comme le réclament certains disciples du second Wittgenstein. $\grave{A}$ l'analyse conceptuelle il revient également, pour Nielsen, de juger de la rationalité de telle ou telle croyance, de déterminer par conséquent la légitimité de telle ou telle idéologie. La philosophie consent à se faire servante de l'idéologie, pourrions-nous dire en paraphrasant Kant, dans la mesure où elle consent à précéder la gracieuse dame avec sa torche et non à marcher à sa suite en portant sa traîne.

On comprend dès lors qu'il ne faille pas chercher dans Contemporary Critiques of Religion un examen détaché des

10. Voir le texte où cet engagement se trouve défini de la façon la plus claire, Religion and Commitment, d'abord publié dans Religious Language and Knowledge, ed. R. H. Ayers and W. T. Blackstone, Athens, Ga., University of Georgia Press, 1971, et reproduit dans N. O. Schedler, Pbilosopby of Religion. Contemporary Perspectives, N.Y., Macmillan, 1974, pp. 387-407.

11. Religion and Commitment, p. 389.

12. Nielsen s'applique à plusieurs reprises à préciser le sens de cette neutralité de la philosophie. Pat exemple, Contemporary Critiques, p. 10 ; «In Defense of Atheism》, in N. O. Schedler, Pbilosopby of Religion, pp. 274-275; Reason and Practice, p. 397. 
principales critiques récentes de la religion. D'abord le but de Nielsen n'est pas de décrire, mais d'arbitrer, de juger si la religion - c'est-à-dire le christianisme, le judaïsme et l'islamisme, qu'il ne tient pas pour des religions supérieures mais pour les seules devant lesquelles il y ait concrètement une décision à prendre est encore viable après les critiques contemporaines auxquelles elle a été soumise ou si plutôt ses droits n'ont pas été par elles totalement ruinés (p. 11). Puis il a seulement en vue ce que j'appellerais les critiques de la validité de la religion, mettant entre parenthèses les critiques de sa genèse. Je m'explique. Nielsen partage la conviction de tous ceux qui, à la suite de Feuerbach, Marx et Freud, croient que les sceptiques du temps des Iumières ont définitivement montré la fausseté de la religion et se donnent pour tâche d'expliquer la persistance de la foi religieuse par les besoins psychiques et sociaux inconscients. C'est la conviction des critiques de la genèse de la religion. Mais Nielsen refuse à bon droit de fonder sur cette conviction sa critique radicale de la religion. Ce serait ignorer qu'il y a des intellectuels pour qui la religion est intelligible et vraie et à qui les critiques de l'Aufklärung paraissent lestées de préjugés injustifiables (p. 1). Il importe donc, selon Nielsen, de mettre entre parenthèses pour un moment la critique de la genèse de la religion et de prendre en mains la critique de sa validité. Ce qui veut dire, puisque c'est un analyste qui ramasse ses outils et s'apprête à se mettre au travail, prélever sur la religion concrète le concept de «Dieu », mettre au jour les principaux arguments développés dans la philosophie analytique pour l'inintelligibilité et l'incohérence de ce concept, puis, en tenant compte des répliques qu'ils ont suscitées, raffiner ces arguments et en arriver à montrer la rationalité - et par conséquent le triomphe - de certaines critiques cruciales de la religion. ${ }^{13}$ Il s'agit donc d'une stratégie qui vise à immuniser la critique de la religion contre toute critique, et si l'on ne craignait pas de compliquer une expression lancée jadis par Guitton, on pourrait lui donner le titre de critique de la critique de la critique de la religion.

Cette stratégie se déploie en trois étapes. La première (chapitres $2,3,4)$ consiste à examiner la critique empiriste de la

13. «I shall try to show that there are crucial contemporary critiques of religion which rest on no mythology 》 (p. 6). 
religion, telle qu'elle fut formulée par l'empirisme logique, à regarder ensuite les attaques auxquelles elle a été soumise, afin de déterminer ce qui de cette critique empiriste résiste à la critique et demeure valable. La thèse de Nielsen est assez claire : l'empirisme logique est mort, il n'est plus possible de professer raisonnablement son dogme fondamental, le célèbre critère de sens suivant lequel, outre les propositions analytiques, seules les propositions vérifiables en principe ont un sens; mais la critique de la religion issue de l'empirisme logique demeure vivante et conserve sa force corrosive. Pourquoi le critère de sens de l'empirisme logique est-il intenable? Avec beaucoup de finesse à mon avis, Nielsen expose les raisons de ce verdict (pp. 32-37). Je retiens les trois principales: d'abord le sens est logiquement préalable à la vérification, car comment peut-on vérifier un énoncé dont on ignore le sens; en outre, ce qui est sensé ou insensé c'est la phrase (sentence), tandis que ce qui est vrai ou faux c'est la proposition (statement), de sorte qu'il peut y avoir sens sans vérification; enfin tout empiriste reconnaît que le critère de vérifiabilité est trop étroit, qu'il y a une foule d'énoncés non vérifiables (ex. : tout livre contient des fautes d'impression) qui sont pourtant dotés de sens (pp. 32-33). Que reste-t-il alors de la critique empiriste de la religion lorsqu'on la dépouille ainsi de son dogme fondamental? Il lui reste, d'après Nielsen, de montrer, indépendamment de tout dogme préalable, le défaut inhérent à tout énoncé sur Dieu : celui de ne se prêter à aucune vérification empirique (experiential confirmation or disconfirmation) (p. 40). Un énoncé sur Dieu, si évocateur soit-il, ne constitue jamais une affirmation authentique et on ne peut jamais lui reconnaître un droit à la vérité parce qu'il n'est jamais possible d'indiquer quels faits ou quels événements pourraient le confirmer ou le réfuter (pp. 39-40).

Ce verdict de Nielsen sur la critique empiriste de la religion, et en particulier sur le triomphe décisif de l'attaque de Flew, aucune des objections dont elle a été l'objet, ni celle d'E. L. Mascall (pp. 40-54), ni celles d'A. Platinga (55-71 et de J. Hick (71-74), ne réussissent à l'ébranler. Il faut dire que Nielsen exécute parfois sommairement ses adversaires. La critique empiriste de la religion passe, selon lui, avec succès le test de ses examinateurs. On peut la revendiquer sans crainte des objections 
qu'elle provoque (p. 92), en étant certain qu'elle ne s'appuie pas nécessairement à une théorie métaphysique du langage et de son rapport au réel (p. 82), et renforcé dans la conviction que le sécularisme n'est pas une conception du monde moins profonde que celle offerte par la religion ou la métaphysique, qu'il a même l'avantage de libérer du besoin de répondre à des questions qui pour le moins semblent absolument insolubles (p. 45).

La deuxième étape de la stratégie (chapitre 5) consiste pour Nielsen à retirer aux analystes croyants leur dernier recours : celui de se réclamer du second Wittgenstein pour soutenir que le langage de la foi est autonome, qu'il doit être compris seulement à partir de ses propres règles et de ses propres mouvements, et qu'il doit par conséquent être soustrait à la critique empiriste de la religion. Ce geste de ceux que Nielsen appelle les «fidéistes Wittgensteiniens » en fait à ses yeux une victime facile et il faut dire qu'il a très bien vu la vulnérabilité de cette forme d'apologie de la religion. C'est peut-être sa contribution la plus importante à la philosophie analytique d'avoir mis au jour les implications du relativisme conceptuel des Philosophical Investigations. L'apologète qui s'en sert pour immuniser le jeu du langage propre à la religion contre toute critique empiriste ne se rend pas compte qu'il prépare en fait la destruction définitive du discours religieux (p. 103). Car si le relativisme conceptuel est vrai, s'il est vrai que l'on est toujours conceptuellement prédéterminé par son contexte linguistique, il faut alors refuser à toute affirmation de la foi un droit quelconque à l'universel. Admettre en même temps le relativisme conceptuel et la vérité des énoncés de la foi (comme le font Holland, Malcolm, Phillips) c'est embrasser l'irrationnel, car c'est prétendre qu'une croyance est vraie sans être plus vraie qu'une autre (p. 109). Nielsen n'a pas de difficulté à montrer que l'acceptation lucide du relativisme conceptuel ne peut qu'impliquer la critique la plus corrosive de la foi et conduire au scepticisme religieux.

Dans la troisième et dernière étape (chapitre 6), la critique de Nielsen se fait plus agressive. Il ne s'agit plus de soumettre les énoncés de la foi aux règles imposées par une philosophie toute faite, ni par conséquent de les juger inintelligibles à partir d'un critère général de sens, mais de montrer au théologien, à l'aide de la seule analyse logique, l'incohérence de ses concepts 
(p. 114). Cette forme de critique de la religion, pratiquée avec brio par plusieurs analystes récents (J. N. Findlay, P. Edwards, B. A. Farrell, R. Hepburn, C. B. Martin, A. Flew), Nielsen cherche à montrer, dans ce chapitre intitulé «On dining with theologians », qu'elle résiste à la contre-critique. C'est dans ce chapitre, à mon avis, que l'analyse conceptuelle opérée par une raison qui se pense mesurée par rien, trahit le plus nettement son attache à une métaphysique, mieux à une ontologie. ${ }^{14} \mathrm{Niel}-$ sen redoute tant l'infiltration d'une ontologie dans son ouvre, qu'il ne peut admettre le besoin de clarifier et de critiquer celle dont il ne peut se passer. Quelle est cette ontologie? Il l'a luimême, dans un autre ouvrage, qualifiée de reductive materialism; elle affirme que tout ce qui existe est corporel. ${ }^{15}$ Doublée d'une

14. Nielsen a exposé ses vues sur la métaphysique dans Reason and Practice, pp. 393-522. Il en distingue trois formes : a) la métaphysique comme effort systématique de connaissance a priori du monde (ibid., p. 438). Cette métaphysique constitue pour Nielsen une ontologie, définitivement ruinée par l'empirisme logicue ( Such ontologizing... is out. It is neither a reasonable nor even a coherent activity. I have argued for such dethronement of ontology. 》Ibid., p. 470). b) La métaphysique comme description de la structure actuelle de notre pensée du monde, remise en faveur parmi les analystes par Strawson. Cette métaphysique, souligne Nielsen (ibid., p. 486), n'implique aucune ontologie; c'est pour cela qu'elle ne met aucunement celui qui la pratique en conflit avec la critique empiriste. c) Enfin la métaphysique comme lecture unifiée de l'expérience (p. 487), comme spéculation contrôlée par la critique rationnelle (p. 517), comme idéologie (p. 521). Nielsen soutient que cette métaphysique est possible et désirable (p. 487), et il en développe une qu'il qualifie de matérialiste (p. 521). Je n'arrive pas à voir comment il puisse méconnaître que sa métaphysique implique une ontologie ( $\varangle$ il n'y a d'être que l'être matériel $\gg$ ), une transgression du pur logique, pourtant combattue sans cesse par lui. C'est ce qui fait pour moi l'irrationnel de sa philosophie. - Notons en passant que Nielsen n'est pas à son meilleur lorsqu'il rapporte le langage des métaphysiciens. Ainsi dans Contemporary Critiques, il fait dire à Thomas d'Aquin que Dieu est « being as being entity itself 》 (p. 130). J'ignore si cette expression a un sens, mais je sais qu'elle se trouve nulle part chez Saint Thomas. Peut-être aurait-il fallu avouer, comme Teilhard le faisait avec bonhomie, que dans la platebande de la métaphysique, il se meut comme un éléphant, ou, comme disent les Anglais, "like a bull in a china shop ».

15. Cette métaphysique matérialiste se trouve développée au chapitre 29 de Reason and Practice, pp. 351-376. Voir également p. 521: «How then should I characterize my ideology? I would describe it as a form of socialist humanism with a materialist metaphysic as its metaphysical backdrop. It is materialist because it rejects all conceptions of deity, Nirvana, of spiritual or mental realities that are not characterizable in purely physical terms, including behavioral terms. » 
noétique radicalement empiriste, qui ne reconnaît à la raison d'autre fonction que celle d'organiser les données de l'expérience en une langue bien faite, cєtte ontologie suffit déjà à imposer au croyant un défi qu'il ne pourra jamais relever et dont il est facile, sans même invoquer l'arsenal d'arguments invoqués en sa faveur, de proclamer le triomphe décisif. C'est seulement à partir de cette ontologie et de cette noétique, me semble-t-il, que Nielsen peut juger irréfutables les principaux arguments des analystes en faveur de l'incohérence du concept de «Dieu ». Ancrée à de tels présupposés, la critique n'a pas de peine à entériner l'athéisme, voire à l'armer de raisons, à lui apprendre par exemple que l'affirmation chrétienne «Dieu est amour » est insensée, puisque le mot «amour» attribué à une réalité incorporelle perd tout son sens (p. 128), et que le concept de «Dieu » est incohérent puisqu'il désigne un référent qu'il est impossible d'identifier empiriquement $\gg\left(\right.$ p. 134). ${ }^{16}$

En présentant ce livre, Nielsen disait avoir pour but, entre autres, de créer un dialogue entre croyants et sceptiques. ${ }^{17}$ Peuton appeler dialogue ce réquisitoire contre la rationalité de la foi, où le fardeau de la preuve repose constamment sur le croyant et où les règles du jeu lui interdisent a priori la possibilité de toute preuve? Le dialogue entre le croyant et l'incroyant ne doit-il pas confronter deux certicudes : celle de l'absurdité de l'existence sans Dieu, celle de l'absurdité de la croyance en Dieu? Dans cette confrontation, admettons que le fardeau de la preuve incombe toujours au croyant, puisqu'il semble bien que l'on puisse s'habituer à vivre sans Dieu et trouver cela parfaitement raisonnable, tandis qu'on ne peut pas s'habituer à croire en Dieu. Pour écouter la preuve du croyant, l'incroyant ne doit-il pas consentir à douter un moment, non pas de la raison, mais du modèle de rationalité qu'il est tenté d'imposer à son partenaire? Ne doit-il pas consentir à renoncer, pour un certain moment, non pas à être raisonnable, mais à vouloir à tout prix avoir raison?

16. Nielsen revient souvent sur l'impossibilité de détecter ou d'identifier Dieu. Voir, entre autres, son article récent, «God and Postulated Entities》, in Soutbern Journal of Philosopby, XII (1974), 225-230.

17. «It will be my aim, by taking certain responses of belief to unbelief, to create what in effect is a dialogue between believers and sceptics.» (Ibid., p. 4). 
Dans son deuxième ouvrage, Scepticism, Nielsen s'applique à définir l'attitude à laquelle conduit la critique contemporaine de la religion (c. 1) et à montrer qu'elle résiste aux essais d'analystes récents pour la surmonter (cc. 2-5). Qu'est-ce que le scepticisme en matière de religion? C'est, soutient Nielsen (p. 4), l'attitude commune à l'athéisme et à l'agnosticisme. L'athée est celui qui rejette la croyance en Dieu parce qu'il croit qu'il est faux ou probablement faux qu'une telle réalité existe, ou parce qu'il croit que le concept de Dieu est si incohérent et si problématique qu'il est impossible d'affirmer de façon intelligible et justifiable qu'il soit vrai ou faux qu'une telle réalité existe, ou enfin parce qu'il croit que le mot «Dieu » désigne seulement une réalité mondaine. L'agnostique est porté à croire ce que croit l'athée, mais il est également enclin à croire le contraire et n'arrive pas à juger ce qu'il est le plus raisonnable de croire en ce domaine (ibid.). Le scepticisme contemporain, ajoute Nielsen (p. 6), comporte un aspect particulier par rapport au scepticisme traditionnel: il n'est pas tellement intéressé à la question de la vérité de la foi religieuse, mais seulement à la question plus fondamentale du sens. Il se fonde sur la conviction que le langage sur Dieu n'a pas de sens, qu'il est incompréhensible, incohérent, inintelligible, et n'offre pas par conséquent une option raisonnable.

Défendre cette nouvelle forme de scepticisme, montrer qu'il résiste à la critique, voilà le but principal de cet ouvrage, qui n'apprend rien de neuf sur l'attitude de Nielsen devant la foi, ni sur la philosophie dont il se sert pour justifier ce qu'il appelle - d'une expression qui étonne de la part d'un penseur si soucieux de vouloir tout soumettre à la vérification empirique - la «pervasive conviction among the intelligentsia that such religious beliefs are incoberent» (p. 39). Trois critiques différentes du scepticisme sont examinées. En premier lieu, celle des fidéistes Wittgensteiniens, que Nielsen pourchasse dans chacun de ses ouvrages, représentés ici par P. L. Holmer et D. Z. Phillips (c. 2), qui tiennent pour faussée toute critique qui impose à la religion des critères d'intelligibilité et de vérité empruntés à une autre forme d'existence. Nielsen n'a pas de peine à montrer encore une fois que ce fidéisme est intenable puisqu'il condamne à tenir pour impossible a priori toute appréciation rationnelle des diverses 
formes de l'existence humaine. Puis vient la contre-critique de ceux qui, tel Mavrodes, en appellent à l'expérience de Dieu. Nielsen admet que s'il y a des hommes qui ont réellement cette expérience de Dieu, il s'ensuit que le concept de Dieu n'est pas incohérent et que le scepticisme se trouve ruiné (p. 42). Mais il n'est pas possible, à ses yeux, de reconnaître à l'expérience de Dieu un droit à la vérité, pour la simple raison que par définition Dieu est une réalité infinie et transcendante et que seule la réalité finie peut en principe être objet d'expérience (p. 47). On voit que le fardeau imposé au croyant par le nouveau scepticisme est déraisonnable: il lui demande l'impossible: adopter une ontologie matérialiste et une noétique empiriste et confesser en même temps l'existence de Dieu. On ne s'étonnera donc pas qu'il juge également inefficaces les essais pourtant remarquables de $\mathrm{N}$. Smart, D. Evans, G. Kaufman, I. T. Ramsey, pour décrire l'expérience religieuse et pour montrer qu'en elle seule le langage de la foi tire son sens (c. 4).

Il ne reste plus à Nielsen qu'à comparer le scepticisme qu'il défend à celui de certains philosophes actuels (N. R. Hanson, S. Hook, W. I. Matson, E. Nagel, M. Scriven), pour qui les énoncés de la foi ne manquent ni de sens ni de cohérence mais sont sans fondement et doivent être rejetés (c. 5). Leur attitude s'explique, pense Nielsen, par l'anthropomorphisme de leur concept de Dieu. Lorsque la critique de la religion porte sur un Dieu représenté comme être fini, elle n'a pas de peine à soutenir que ce concept est intelligible mais sans fondement. Mais ce genre de critique ne rend pas justice à la religion, car le croyant réfléchi récuse un tel concept; en outre, elle péche par pétition de principe puisque ce qu'elle déclare sans fondement c'est autre chose que le concept religieux (p. 93). La critique pratiquée par Nielsen se veut innocente de cette faute, aussi prend-elle soin de ne jamais s'arrêter à une vue anthropomorphique de Dieu.

Le critique de la religion s'impose une dernière tâche avant de déclarer son travail fini: il lui faut montrer que le départ de Dieu n'a aucune importance pour la qualité de la vie morale, que ce qui apparaît au croyant comme le déclin de la sensibilité religieuse et n'est en fait que la certitude croissante de l'absurdité de la religion (p. 102) n'entraîne nullement la désorientation de 
l'existence humaine (p. 100). C'est à cette tâche que s'attaque Nielsen dans un petit livre qui ne manque pas de virtuosité logique, Ethics without God.

Au début de la lecture de ce livre, je crus qu'il me serait possible, beaucoup plus que dans les précédents, de faire route avec l'auteur. Un discours sur l'éthique, me disais-je, a l'avantage de porter sur quelque chose de commun à tous, préalable à toute division idéologique: la recherche d'une raison de vivre. Ici, je l'espérais, on ne sera plus gêné par un modèle étroit de rationalité puisqu'il ne s'agit plus de juger de la cohérence du langage mais d'expliciter la logique de la vie. Une surprise me guettait: celle de constater qu'un analyste, pourtant si soucieux de toujours travailler avec des concepts clairs, si habile à dépister les moindres indices de «muddle», peut tolérer généreusement des notions restées implicites et confuses lorsqu'il déploie sa philosophie pratique. Qu'est-ce que la morale dont on soutient, à bon droit, la primauté par rapport à la religion? Rien de bien précis, sinon un système de contrôle social inventé par l'homme pour protéger ses intérêts. ${ }^{18}$ Qu'est-ce que l'idée du bien, qu'on affirme, à bon droit encore, indépendante de l'affirmation de Dieu? Est bon, semble-t-il, tout ce qui peut être source de bonheur (p. 56). Qu'est-ce que le bonbeur dans la recherche duquel on voit, à bon droit toujours, le ressort de la vie morale? Ce que tout le monde désire, bien sûr, c'est-à-dire la libération de la souffrance et la satisfaction d'un ensemble de besoins fondamentaux de la vie (p. 53).

C'est avec ces concepts à peine dégrossis, que Nielsen se met à défendre trois thèses, toutes visant à couper le lien ombilical de la moralité avec la religion. La première consiste à montrer l'erreur du croyant qui voit dans la volonté de Dieu le critère dernier de la moralité (c. 1). Pour se soumettre à la volonté de Dieu, soutient Nielsen, le croyant doit estimer Dieu parfaitement bon. Mais il ne peut estimer Dieu parfaitement bon sans au préalable comprendre ce que c'est qu'être bon. La proposition «Dieu est bon», qu'elle soit synthétique ou, ce qui est plus vraisemblable, analytique, n'est possible qu'à celui qui déjà,

18. Voir Reason and Practice, p. 308. 
indépendamment de sa connaissance de Dieu, possède une idée du bien. Cela suffit à démontrer, toujours selon Nielsen, non seulement que la morale est indépendante de la religion, mais qu'elle en est le nécessaire fondement (pp. 7-14).

Je ne contesterai sûrement pas que l'idée du bien soit logiquement préalable à l'affirmation de la bonté de Dieu, comme d'ailleurs l'idée d'être est logiquement préalable à l'affirmation de l'existence de Dieu, et je trouve dommage que Nielsen ne le reconnaisse pas, car, à y penser, on comprend qu'aucune affirmation de l'existence ou de l'inexistence de Dieu n'est tout à fait libre d'une ontologie. Ce que je contesterai, c'est la façon de poser le problème du rapport entre religion et moralité, viciée au départ par des notions confuses, mais surtout par l'absence d'une distinction indispensable à l'intérieur de la réalité concrète d'une religion (judaïsme, christianisme, islamisme) entre la foi et la religion elle-même. ${ }^{19}$ Autant la vie morale est indépendante de la religion, autant elle est impensable sans une foi, sans l'adhésion à une raison de vivre, à une idée de l'homme à réaliser : que je veuille la tirer de moi seul ou que j'accepte à son sujet de me laisser enseigner par le Créateur.

La seconde thèse d'Ethics without God consiste à dire que la morale, pour être authentique et adéquate, n'a pas besoin de Dieu (c. 2). On peut être porté à croire que sans Dieu la vie humaine n'a pas de but, mais "ne pas avoir de but », écrit Nielsen, a un double sens: ne pas être soumis à une fin et ne pas avoir de raison de vivre. L'homme en tant qu'homme, soulignet-il, n'a pas de but, si l'on entend par but une fin qui lui serait imposée par la nature. Penser que l'homme est fait pour quelque chose c'est offenser sa dignité, le ravaler au rang des choses. Cela ne veut cependant pas dire que l'homme soit sans but, au sens de raison de vivre (38-41). Qu'il n'y ait pas de but de la vie, affirme Nielsen, n'implique pas qu'il n'y ait pas de but dans la

19. Voir sur ce point $P$. RICGEUR, «Sciences humaines et conditionnements de la foi $»$, in Semaine des intellectuels catboliques, 1965, p. 139 ss; M.-D. CHENU, "Foi et Religion», in Les Études philosopbiques, 21 (1966), 357-369. 
vie. ${ }^{20}$ On voit l'humanisme pour lequel Nielsen opte résolument : l'homme est quelque chose à dépasser ${ }^{21}$, - sometbing to be surpassed; parce qu'il est sans raison, il est seul responsable de ses raisons de vivre. Admettre une prétendue fin de l'homme, ce serait renoncer à la vue scientifique du monde (p. 40). Ne faudrait-il pas, comme Sartre, avouer la vraie raison : il n'y a pas de nature humaine parce qu'il n'y a pas de Dieu pour la penser. Mais c'est gênant de faire cet aveu, car on n'est pas loin d'admettre qu'on se meut dans un cercle dans lequel on peut certes sauter mais sans pouvoir jamais en sortir. Car n'affirme-t-on pas qu'il n'y a pas de Dieu parce que le monde et l'homme dans le monde sont tels que rien ne permet de le penser?

Comme troisième et dernière thèse, Nielsen fait l'apologie de l'éthique de l'humanisme athée, dont la qualité n'est pas inférieure, à ses yeux, à celle des éthiques religieuses (c. 3). Il s'agit d'un chapitre plutôt faible où Nielsen essaie de montrer que l'humanisme athée offre un modèle d'existence qui conjugue la recherche du bonheur et le souci de soulager la souffrance, qui retient, pourrait-on dire, tout ce qu'il y a de positif dans l'éthique chrétienne en l'affranchissant de ce qui constitue le crucifiement de l'intellect: croire que nous sommes créatures de Dieu (p. 60). À comparer ainsi, comme le fait Nielsen, le christianisme ou le judaïsme comme réalités sociologiques séculaires, avec une réalité à venir - l'éthique humaniste - ne se donne-t-il pas un moyen facile d'avoir raison? C'est un procédé qui étonne de la part d'un empiriste. Lorsqu'il dit, par exemple, que devant la souffrance humaine seul le croyant cherche à la justifier tandis que l'humaniste lui la combat avec courage et dignité, n'a-t-il pas donné congé au principe de falsifiabilité ? On a beau ne pas reculer devant le blasphème, peut-on sincèrement oublier que le fondateur de la religion chrétienne n'a jamais tenté d'expliquer le mal et en a résolument assumé les conséquences, sans attendre de les avoir parfaitement calculées?

20. «People who claim that if God is dead nothing matters constantly conclude that there can be no purpose in life because there is no purpose of life... Ethics without God, pp. 40-41. Voir également Reason and Practice, p. 255. " Man need not be made for a purpose in order for life to have a purpose and a point. »

21. Voir Reason and Practice, p. 271. 
Le dernier chapitre d'Etbics without God est un petit essai de casuistique utilitariste, fort révélateur, à mon avis, de la myopie de l'éthique professée par Nielsen. La morale utilitariste la seule sans doute possible à un matérialiste conséquent prescrit de choisir dans les différentes situations le genre d'action qui entraîne le moins de souffrance et le plus de bonheur pour tous (p. 77). Il peut lui arriver de contredire lẹ bon sens, de prescrire par exemple le meurtre d'un innocent pour éviter la souffrance d'un plus grand nombre. Qui se fera le juge des choix à faire dans chaque cas? La conscience individuelle? Il ne semble pas. Le meilleur juge de chaque situation sera le mieux équipé pour prévoir toutes les conséquences de chaque geste humain. Ne sommes-nous pas devant une éthique qui a besoin de s'appuyer sur l'autorité d'un parti ou d'un Fuehrer, d'un philosophe ou d'un technocrate? Nielsen avoue dans sa Préface que son épouse n'est pas d'accord avec la direction prise par ce dernier chapitre. Elle exprime à mon avis le simple bon sens.

On me permettra, au terme de cette analyse des trois livres de Nielsen, d'exprimer un regret. Son œuvre se présente comme la reprise de la critique de l'époque des lumières. Il veut s'assurer, avant de s'occuper de critiquer la genèse de la religion, c'est-à-dire avant de s'apprêter à décomposer la religion pour l'expliquer, que la critique de sa validité est achevée, qu'elle résiste à toute contrecritique. Je regrette cependant que Nielsen n'accorde aucune attention aux raisons qu'avait Hegel contre la critique de l'Aufblärung dans laquelle il a vu un échec monumental. Qu'on le veuille ou non, Hegel a adressé aux rationalistes un défi qui n'est pas moins sérieux que celui de Flew aux théologiens: celui de prouver qu'une critique de la pure validité de la religion, c'est-àdire une critique qui ne se donne pas pour norme le contenu même de la religion, puisse éviter de réduire ce contenu aux préjugés de l'entendement. C'est trop facile, me semble-t-il, d'aller de Kant à Marx, sans passer par Hegel. C'est en tout cas rater l'occasion de comprendre que la pensée philosophique s'impose, à certains moments, de développer une ontologie. C'est prendre le risque également de ne jamais rencontrer le vrai Marx. 


\section{T. Penelhum et la neutralité de la philosophie de la religion}

Avec les travaux de T. Penelhum ${ }^{22}$, la philosophie analytique déploie pour l'étude de la religion une autre possibilité: celle du maintien de la stricte neutralité. ${ }^{23}$ En quoi consiste cette neutralité ? Elle est d'abord une règle méthodologique que le philosophe de la religion doit s'imposer: faire en sorte que son analyse soit indépendante de son attitude face à la vérité de la foi religieuse. ${ }^{24}$ Il s'agit, au concret, de renoncer au fidéisme Wittgensteinien ${ }^{25}$, de ne jamais immuniser le discours de la foi contre la critique en soutenant que le sens de ce discours n'est accessible qu'à celui qui s'engage à l'égard de sa vérité. Le point de vue de la neutralité consiste donc à ne pas présupposer la foi, à ne pas supposer que le sens de son discours soit inaccessible à l'incroyant, voire à ne pas exclure a priori que dans ce discours il puisse y avoir du non-sens ou de la contradiction.

Cette neutralité philosophique, adoptée au départ comme simple règle méthodologique, deviendra chez Penelhum, à mesure que se développera son analyse de la foi, la thèse capitale de sa philosophie de la religion et la distinguera non seulement du fidéisme Wittgensteinien mais aussi du scepticisme de certains analystes. Quelle sera cette thèse? Celle de l'impuissance de la philosophie à juger de la vérité de la foi religieuse et à se faire l'arbitre entre la foi et l'incroyance. L'analyste peut montrer que la foi n'est pas irrationnelle, que les arguments invoqués pour prouver son inintelligibilité ou sa contradiction interne peuvent être réfutés, mais il ne peut pas décréter la foi si raisonnable que

22. Religion and Rationality. An Introduction to the Pbilosopby of Religion, N.Y., Random House, 1971, 392 p.; Problems of Religious Knowledge, London, Macmillan, «Philosophy of Religion Series », ed. by J. Hick, 1971, 186 p.; Survival and Disemtodied Existence, London, Routledge and Kegan Paul, «Studies in Philosophical Psychology», ed. by R. F. Holland, 1970, $111 \mathrm{p}$.

23. Penelhum développe cette thèse de la neutralité philosophique dans Religion and Rationality, p. XI, p. 202 ss; p. 355, et dans Problems of Religious Knowledge, pp. 1-7; p. 119 ss.

24. Problems of Religious Knowledge, p. 2.

25. Dans «Logic and Theology 》, in Canadian Journal of Theology, 4 (1958), 255-265, Penelhum montre que si la philosophie analytique post-wittensteinienne n'est plus essentiellement sceptique, comme l'était l'empirisme logique, le langage théologique n'est pas pour autant soustrait à sa critique. 
l'incroyance serait absurde, ni prouver par conséquent que le scepticisme est sans raison et ne constitue pas une alternative rationnellement justifiable.

C'est à la défense de cette thèse qu'est consacré le premier ouvrage de Penelhum, Religion and Rationality. Dans la première des trois parties (cc. 1-15), il s'attaque à une tâche préliminaire: déblayer le terrain longtemps occupé par la théologie naturelle, qui représente pour la raison philosophique un rapport possible avec la foi - celui-là même que l'on identifie à l'œuvre de Thomas d'Aquin - et qu'il faut absolument réfuter pour rendre à la philosophie de la religion sa fonction purement analytique. Car le pratiquant de la théologie naturelle, du moins tel que Penelhum le comprend, se croit en mesure de démontrer certaines vérités de la foi - l'existence de Dieu, sa nature, son rapport avec le monde - et de poser le sceau de la rationalité sur l'ensemble du discours de la foi, c'est-à-dire de le décréter raisonnable au sens étroit de démontrable ou scientifiquement probable et de juger insensée ou contradictoire l'incroyance (p. 3).

Où réside pour Penelhum l'erreur de la théologie naturelle? D'abord dans le fait qu'aucune de ses démonstrations de l'existence de Dieu ne résiste à la critique. La preuve ontologique repose sur une erreur conceptuelle sur l'existence qui ne peut jamais faire partie d'un concept, même celui de Dieu (p. 17); la preuve cosmologique des célèbres cinq voies de saint Thomas se réduit finalement à la preuve ontologique puisqu'elle affirme un être dont la nature contient la raison d'être de son existence (p. 38) ; la preuve téléologique, la moins géniale et la plus faible de toutes, et pourtant celle que l'on abandonne le moins facilement (pp. 54-55), propose une hypothèse explicative du monde que l'on a tort d'estimer nécessaire. Ensuite, une autre erreur de la théologie naturelle, aux yeux de Penelhum, c'est de confondre indûment deux recherches: celle d'une explication globale du monde; propre à la métaphysique, et celle d'un objet adéquat du culte, propre à la religion (p. 4). On voit ce qui en résulte: une métaphysique, d'une part, qui s'alimente à une vue religieuse du monde et dont les arguments valent seulement pour le croyant, grâce à son adoption préalable du point de vue théiste; une religion, d'autre part, qui emprunte à la raison philosophique son objet: l'être nécessaire, immuable, transcendant. Telle est, 
aux yeux de Penelhum, la confusion inhérente du concept de théologie naturelle.

Faisons une pause devant cette image de la théologie naturelle que Penelhum attribue à saint Thomas, dont il rejette, dans les neuf premiers chapitres de Reason and Rationality, la façon de comprendre le rapport entre la foi et la réflexion philosophique. Cette image, il the faut le dire, ressemble bien peu à ce que fut pour l'Aquinate la théologie philosopbique. À cette théologie, il assignait un rôle bien modeste par tapport à celui de la theologia sacrae scripturae. ${ }^{26}$ Il lui refusait même d'avoir Dieu pour objet et d'en parler autrement qu'in obliquo, c'est-à-dire seulement comme principe explicatif de son objet: l'existant. Cela peut étonner, mais la théologie philosophique de saint Thomas entretenait à l'égard du divin ou du sacré moins de prétention que la plupart des philosophies de la religion. ${ }^{27} \mathrm{Ce}$ qu'elle interrogeait, c'était seulement l'être des choses du monde ; ce qu'elle visait à expliquer, c'était le changement, la contingence, l'ordre, qui caractérisent cette manière d'être. Si elle parvenait à poser l'existence de l'Ipsum Esse, ce n'est pas l'analyse du concept de Dieu qui l'y conduisait, mais la seule lecture de l'être contingent. Juger invalide cette démarche, sous prétexte qu'elle affirmerait l'existence nécessaire du contenu d'un concept, c'est prou-

26. Voir In Boetbium de Trinitate, q.5, a.4, c.; Ibid., q.2, a.2, c.; q.5, a.1, c.

27. Plusieurs analystes récents préfèrent le nom de Théologie philosopbique à celui de Pbilosophie de la religion. Par exemple : ROSS, J. F., Pbilosopbical Theology, Indianapolis, Bobbs-Merrill, 1969; MILLER, E.D.L., God and Reason: a Historical Approach to Pbilosopbical Theology, N.Y., Macmillan, 1972 ; MAVRODES, G. I., "Some Recent Philosophical Theology », in Rev. Met., 24 (1970), 82-111; NIELSEN, K., \& The Primacy of Philosophical Theology », in Theology To-day, 27 (1970), 155-169. Ce n'est pas à saint Thomàs qu'ils l'empruntent. C'est plutôt la préface d'A. Flew et A. MacIntyre aux New Essays in Pbiiosopbical Theology, London, SCM Press, 1955, qui semble avoir répandu cet usage. Le motif de Flew et MacIntyre pour abandonner le titre de Pbilosopbie de la religion et adopter celui de Théologie pbilosopbique est précisément de rompre totalement avec ce qu'évoque à leurs yeux la Pbilosopbie de la religion, à savoir l'entreprise - d'inspiration idéaliste à leur avis - de démonstration des préambules de la théologie théiste (ibid., p. VIII). Que sera leur tbéologie pbilosopbique? Non pas un discours sur Dieu, mais sur le concept de Dieu, d'où, à leur avis, la possibilité, de parler sans pléonasme d'une théologie théiste ou sans contradiction d'une théologie athée. Nous sommes loin de la theologia philosopbica. 
ver qu'on est incapable de lire une œuvre philosophique dont les postulats ne sont pas ceux de Hume ou de Kant. Je respecte la décision de l'analyste de refuser à l'intelligence humaine de comprendre l'être des choses; mais j'aimerais qu'en lisant saint Thomas, qui est incompréhensible sans ce présupposé noétique, et qu'en critiquant sa théologie, on tienne compte de son ontologie. Autrement, ce qui arrive - et c'est bien ce que fait Penelhum - on lit ses preuves de l'existence de Dieu comme si elles avaient pour prémisses: l'énoncé d'un fait d'expérience ( $\ll$ le monde est contingent ») et le principe leibnizien de raison suffisante - qui n'a pas sa place dans l'ontologie thomiste ${ }^{28}$ - pour démontrer qu'il doit y avoir un être dont l'essence est la raison d'être de son existence. Contre cette démarche, qui n'est pas celle de saint Thomas, le critique a beau jert, surtout s'il procède d'une ontologie qu'il ne sent le besoin ni de clarifier ni de justifier, tant il est entendu depuis Kant que l'être est inaccessible à la connaissance humaine.

L'ontologie de saint Thomas fut l'ontologie d'un théologien, bien sûr. Son intention, cependant, en la déployant, n'a pas été, malgré ce qu'en pense Penelhum, de démontrer qu'il est absurde de ne pas croire, ni de fournir au culte un objet acceptable pour la raison. Son intention a été de surmonter le psychologisme dont la foi est menacée lorsqu'e!le en reste au langage de l'invocation, de chercher par conséquent à préserver le mystère de tout anthropomorphisme en interrogeant l'être même de Dieu. Pour dévoiler -l'intentionnalité de cette théologie de style ontologique, peutêtre faut-il se livrer à autre chose qu'à une «piecemeal analysis» de quelques échantillons prélevés aux écrits de saint Thomas et consentir à une lecture patiente de son œuvre, - sans transgression de la précieuse neutralité.

28. Dans Reason and Rationality, pp. 24-25, Penelhum voit dans la preuve soi-disant cosmologique de saint Thomas l'emploi du principe de raison suffisante et, pour clarifier ce principe, en appelle au texte classique de la Monadologie de Leibniz. Bien que la plupart des thomistes aient accordé une place à ce principe dans l'axiomatique de saint Thomas, il semble bien qu'il s'agisse d'un postulat né avec le rationalisme moderne. Il n'est pas impossible à l'analyse de ce principe de montrer qu'il n'est pas évident, voire que c'est sa négation qui est évidente, autrement dit qu'il est absurde. Voir R. VERNEAUX, «Note sur le principe de raison suffisante», dans La Crise de la raison dans la pensée contemporaine, cahier $\mathrm{V}$ des Recherches de Pbilosophie, Paris, Desclée de Brouwer, 1960, pp. 39-60. 
Une fois l'arbre de la métaphysique déraciné de son jardin, le philosophe de la religion peut librement se résoudre à ne cultiver rien d'autre que l'analyse. L'échec de la théologie naturelle a pour effet de limiter la philosophie de la religion à la clarification de ses concepts (p. 87). C'est déjà de cette façon qu'elle était comprise de la tradition augustinienne et anselmienne, avec laquelle la philosophie analytique se reconnaît plus d'affinité qu'avec la tradition thomiste, même si elle refuse de pratiquer l'analyse dans la foi et pour la foi (pp. 201-202). À quelle tâche cette analyse doit-elle s'appliquer? $\bar{A}$ examiner d'abord la nature et le statut du discours religieux afin de déterminer s'il résiste à la critique du sens déployée par le sceptique (cc. 10-15), puis à examiner les principaux arguments des sceptiques visant à montrer la contradiction interne de la foi religieuse (cc. 16-18), enfin à expliciter comment il faut interpréter certains énoncés de la foi afin d'éviter leur conflit avec le savoir scientifique et la connaissance morale (cc. 19-24).

Que révèle l'examen du statut du discours religieux? Il fait voir que la critique n'a réussi à démontrer ni son incohérence, ni son inintelligibilité, ni par conséquent l'irrationnalité de la foi. Il n'est pas incohérent, estime Penelhum, qui illustre sur ce point la position des théologiens de droite devant le défi de Flew, parce qu'il passe le test de la vérification, sans que l'on ait le besoin de nier que les énoncés de foi soient de vraies assertions ou qu'ils puissent être vérifiés (pp. 147-148). Mais pour être vérifiable le discours religieux doit d'abord être intelligible. L'est-il ? Poser cette question, pour Penelhum s'inspirant ici de Strawson, c'est à propos des énoncés de foi demander si leur sujet est identifiable et jusqu’à quel point il taut soustraire de leurs prédicats les limites inhérentes à la façon ordinaire de les attribuer à d'autres sujets. Sa réponse montre bien, à mon avis, que l'outil logique dont se sert son analyse n'a pas été taillé pour scruter le langage de la foi et n'a pas la souplesse nécessaire à l'élucidation de son sens. Il oblige en effet Penelhum à imposer à la foi, pour pouvoir se conformer aux canons de l'intelligibilité, deux conditions : a) cesser de penser Dieu comme un être intemporel et lui reconnaître une vie mentale analogue à la nôtre, autrement ce que l'on attribue à Dieu n'est pas intelligible (p. 192) ; b) cesser également de penser qu'il puisse y avoir d'autres êtres incorporels 
que Dieu, car autrement on ne peut pas identifier Dieu ; quelqu'un n'est identifiable que par son corps et Dieu seulement par son action sur l'univers corporel ; admettre l'existence d'autres êtres incorporels, c'est se rendre incapable d'établir l'identité de Dieu (pp. 156-157). S'il ne se plie pas à ces deux conditions, «l'emploi dans le discours religieux du concept d'un dieu personnel est vide, malhonnête, ou incohérent » (p. 161).

Pareille demande paraîtra étrange et difficilement intelligible à celui qui n'est pas entraîné à la pratique de l'analyse conceptuelle. Il lui faut se rappeler que pour la tradition à laquelle appartient la philosophie analytique le concept n'a pas de visée ontologique. Il n'est pas un verbe formé par l'intelligence pour signifier ce qu'il en est de l'être; il est une simple représentation, mesurée par rien d'autre que par les données sensibles de l'expérience. Il n'est pas étonnant dès lors que l'analyse du concept de Dieu, entreprise sans doute pour dépasser le psychologisme de la foi, soit inspirée par le souci, non pas de l'être de Dieu, qui caractérise la théologie de style ontologique, mais du maintien d'une représentation aux contours suffisamment précis pour lui permettre un tôle dans le discours. Ce qui étonne, c'est que l'analyste, qui se scandalise de la preuve ontologique, fasse lui-même dépendre l'essence de Dieu de notre façon de le représenter. Ce que révèle en tout cas l'analyse, c'est, à mon avis, que le dépassement du psychologisme de la foi vers le logique ne suffit pas, car le logique qui n'est pas lui-même dépassé vers l'ontologique ne fait qu'entériner un autre psychologisme, à peine moins particulier que le premier.

Moyennant les accommodements prescrits, la foi religieuse résiste donc, pour Penelhum, à l'accusation d'incohérence ou d'inintelligibilité. Elle représente une option raisonnable, bien que pas la seule, car le sceptique en refusant l'interprétation théiste du monde ne commet aucune faute contre la logique et s'évite même des complications intellectuelles (p. 210). Que dire maintenant de l'autre grief de la critique, accusant le discours religieux d'entretenir des contradictions entre certains énoncés de foi ?

Penelhum s'attaque d'abord, dans les deux premiers chapitres de la dernière partie (cc. 16 et 17), à la contradiction apparente entre la foi en un Créateur très bon et tout-puissant 
et l'existence du mal dans le monde, et n'a pas de peine à montrer que l'accusation ne tient pas. Il estime plus difficile de concilier la foi à un Dieu immuable et la création du monde dans le temps (c. 18). Il y a, bien sûr, la solution thomiste, mais elle repose, dit-il, sur deux grosses erreurs : celle de verser dans le panthéisme, puis celle de s'appuyer à une notion inacceptable de Dieu comme l'Être lui-même (p. 259). Pour éviter la contradiction, il faut, selon Penelhum, réaménager notre concept de Dieu, corriger certaines perfections traditionnellement attribuées à Dieu : son immutabilité d'abord, car il faut admettre en Dieu une vie mentale déroulée dans le temps, ce qui ne l'empêche pas d'être sans changement comme l'est une personne constante (pp. 254-260) ; son omniscience ensuite, nécessairement limitée par la liberté de certaines de ses créatures (pp. 260-261) ${ }^{29}$ son impassibilité enfin, pour la simple raison que supposer Dieu impassible, c'est prétendre qu'il soit plus honorable d'être sans passion (pp. 261-262).

Il n'y a pas seulement le besoin de concilier entre eux certains énoncés de foi qui inspirent à Penelhum de réinterpréter la croyance en Dieu; il y a encore le besoin d'éviter le conflit entre cette croyance et la connaissance scientifique du monde. Les sept derniers chapitres de Reason and Rationality visent à répondre à ce besoin par l'analyse des miracles (c. 19), de la prière de demande (c. 20), du rapport entre la connaissance divine et la liberté humaine (c. 21), entre la religion et la morale (c. 22), entre le théisme chrétien et la nature humaine (c. 23), et finalement de la survie de l'homme (cc. 24 et 25 ). Après avoir montré que la foi religieuse n'est ni incohérente ni inintelligible, qu'elle ne comporte pas non plus de contradiction interne, il peut maintenant ajouter qu'elle n'entre pas en conflit avec la connaissance scientifique du monde et conclure qu'elle est raisonnable, non pas au sens étroit où elle constituerait la seule option possible, mais au sens large d'option qui n'est pas déraisonnable (p. 355). Voilà la conclusion de cette analyse de la rationalité de la religion, qui accorde son placet à la foi religieuse à condition qu'elle consente à une relecture anthropomorphisante de son concept de Dieu.

29. Le chapitre 21 de Reason and Rationality est consacré à la défense de cette thèse. 
L'un des problèmes les plus difficiles posés par la foi religieuse - problème dont la lecture athée du monde ne s'embarrasse pas - est celui de l'immortalité et de la résurrection. L'analyste de la religion, pense Penelhum, ne peut pas le contourner. Non seulement le concept de survie est-il essentiel à la foi chrétienne, mais s'il est absurde, la thèse de la vérification eschatologique, habilement développée par J. Hick en réponse au défi de Flew n'a plus de chance d'être valide. C'est pour cela que Penelhum est revenu à plusieurs reprises sur ce problème et lui a consacré entre autres un petit ouvrage, Survival and Disembodied Existence. ${ }^{30}$

L'analyse de ce problème vient très près de le conduire à juger irrationnelle la foi religieuse. La doctrine de l'immortalité de l'âme ou de la survie d'un esprit désincarné lui parait tout simplement absurde, pour la simple raison qu'il manque entre l'homme avant la mort et l'esprit désincarné la continuité corporelle, seul critère nécessaire et suffisant d'identité personnelle (p. 76). ${ }^{31}$ Est-ce à dire que l'existence d'un dieu incorporel personnel soit également absurde? Non, pense Penelhum, mais à condition qu'il soit l'unique être incorporel, repérable par son action dans le monde. Admettre une pluralité d'êtres incorporels serait, aux yeux de Penelhum, incohérent: ou bien il n'en existe qu'un ou bien il n'en existe pas du tout (p. 108).

Quant à la doctrine de la résurrection, elle est concevable (p. 92), logiquement viable (p. 99), intelligible (p. 103), mais Penelhum ne voit pas comment on peut l'interpréter sans avoir recours à l'impossible hypothèse de l'existence d'un esprit désincarné qui garantirait la continuité entre le vivant ante mortem

30. Outre Survival and Disembodied Existence et les deux derniers chapitres de Reason and Rationality, deux articles de PENELHUM: «Personal Identity, Memory and Survival 》, in Journal of Pbilosophy, 1959, pp. 882-903; «Personal Identity», in The Encyclopedia of Pbilosophy, ed. P. Edwards, 1967, Vol. 6, pp. 95-107, discutent du problème de l'immortalité. Ajoutons que Penelhum a également édité dans la collection Basic Problems in Pbilosopby Series l'anthologie: Immortality, Belmont, Calif., Wadsworth Publishing Co., 1973, 162 p. On trouvera une intéressante discussion de la thèse de Penelhum dans Robert YOUNG, «Professor Penelhum and the Resurrection of the Body ", in Religious Studies, 9 (14), 181-187.

31. Penelhum qualifie d'absurde cette doctrine dans Religion and Rationality, p. 354. 
et l'être ressuscité (p. 99). Si elle ne rencontre pas de difficulté logique insurmontable, la croyance en la résurrection est pourtant déconcertante (p. 354); elle offre une façon possible de répondre à la question de la survie, mais n'exclut pas la rationalité d'autres interprétations possibles.

Je ne m'arrêterai ni à reproduire ni à discuter l'argumentation de Penelhum en faveur de ces thèses, afin d'accorder plus d'espace à ce que j'estime ètre son meilleur ouvrage, Problems of Religious Knowledge. ${ }^{32}$ Il s'agit d'un essai d' «épistémologie de la religion» (p. 8), non pas au sens où la pratiquent certains analystes, dans le but de déterminer si oui ou non la foi religieuse a droit au titre de «knou'ledge $;^{33}$ ce genre d'épistémologie religieuse, aux yeux de Penelhum, est impossible, puisqu'elle fait nécessairement appel à des critères dont le choix implique une attitude soit croyante soit sceptique (p. 126). Celle que Penelhum $a$ en vue consiste seulement à rendre explicite le désaccord entre le croyant et l'incroyant, c'est-à-dire à clarifier les présupposés et les implications de leur position respective, non pas dans le but de surmonter ce désaccord - il est pratiquement insurmontable, aux yeux de Penelhum - mais pour le vivre aussi intelligemment que possible, avec pleine conscience des limites au-delà desquelles l'accord entre le croyant et l'incroyant n'est plus possible et le désaccord s'instaure irrémédiablement.

Dans la description de Penelhum, le désaccord comporte une conviction commune au croyant et à l'incroyant: celle de l'impossibilité de prouver l'existence de Dieu. C'est dire que le pratiquant d'une théologie philosophique de style thomiste se

32. Bien que Problems of Religious Knowledge et Religion and Rationality aient été publiés tous deux en 1971, il semble que le premier ait été écrit après le second, car il $\mathrm{y}$ renvoie fréquemment. Problems of Religious Knowledge semble également avoir bénéficiẻ de meilleures conditions de travail que Religion and Rationality rédigé durant une période où l'auteur était doyen de la Faculté des Arts à Calgary. Notons enfin que Penelhum a donné une première version abrégée des chapițres 2 et 3 de Problems of Religious Knowledge sous le titre significatif «Is a Religious Epistemology Possible? 》 in Knowledge and Necessity, Royal Institute of Philosophy Lectures, Vol. III, London, Macmillan, 1970, 263-280.

33. Comme exemple de ce genre d'épistémologie, W. T. BLACKSTONE, The Problems of Religious Knowledge. The Impact of Philosopbical Analysis of Religious Knowledge, Prentice-Hall, Englewood Cliffs, N.J., 1963, 175 p. 
trouve exclu de cette confrontation. C'est ce qui fait, pour une bonne part, l'intérêt du livre: de faire voir, avec beaucoup de lucidité, ce qu'implique cette conviction, notamment l'impossible communication entre la foi et l'incroyance. Le croyant, qui estime impossible de prouver l'existence de Dieu, se voit obligé, explique Penelhum, d'appuyer son adhésion aux énoncés de foi sur une sorte de conscience immédiate de Dieu et d'instituer par là une séparation épistémologique radicale d'avec l'incroyant, du fait qu'il en appelle à une prétendue expérience cognitive que l'incroyant, qui en est privé, soupçonne d'être fausse. En déployant ainsi la logique de cette conviction présupposée par le désaccord entre croyant et incroyant, Penelhum en arrive à soutenir qu'il s'agit d'un présupposé qu'ils devraient refuser de tenir pour évident et dont ils devraient reconnaître qu'il repose sur un fondement contestable (p. 148).

La première étape de Problems of Religious Knowledge est consacrée à l'analyse du concept de preuve et de son application aux énoncés de foi (cc. 2 et 3 ). Il s'agit de clarifier le présupposé, commun au croyant, et à l'incroyant, de l'impossibilité de prouver un énoncé de foi et d'en dégager les implications. Défini plus rigoureusement, ce présupposé, auquel Penelhum donne le nom de «non-naturalisme théologique 》 (p. 54), signifie l'impossibilité d'inférer une conclusion théiste de prémisses non théistes, c'est-à-dire de prémisses qu'on peut tenir pour vraies sans savoir que Dieu existe (p. 52). Ce principe épistémologique, ajoute Penelhum, est susceptible de deux interprétations: l'une, modérée, se limite à affirmer l'impossibilité de fait de trouver des propositions non théistes dont on peut inférer l'existence de Dieu ; l'autre, radicale, soutient leur impossibilité de droit (p. 60). Or le non-naturalisme théologique radical constitue, pour Penelhum, une position absurde. Il est donc en principe possible au croyant et à l'incroyant de se mettre d'accord sur le genre de prémisses non théistes qui réussiraient à libérer une conclusion théiste de tout doute raisonnable, mais il est pratiquement impossible de décider de cés prémisses sans s'appuyer sur des critères préalables, de la foi ou de l'incroyance. Devant une preuve invoquée par le croyant pour fonder ses énoncés de foi et estimée valide par lui, le sceptique peut toujours raisonnablement contester que les prémisses de cette preuve soient vraies ou soient 
connues comme vraies (p. 64).

L'analyse de la vérification des énoncés de foi (c. 4) conduit à la même conclusion. Encore ici, Penelhum juge arbitraire et absurde l'interprétation radicale du non-naturalisme (p. 76) et plus arbitraire encore, parce que plus radical, le positivisme théologique des vérificationnistes (pp 77-78). Encore ici, il teconnaît qu'en principe, il est possible au croyant et à l'incroyant de s'entendre sur ce que l'on pourrait concevoir comme servant à vétifier le discours religieux et l'exprimer en un langage non théiste. Penelhum prétend mème que la thèse de Hick sur la vérification eschatologique fournit un exemple de procédé possible de vérification en principe admissible par le croyant et l'incroyant (pp. 80-81). Mais, de fait, le croyant et l'incroyant n'accordent pas la même vraisemblance à l'affirmation des événements eschatologiques, et leur différence d'attitude vient uniquement de la présence chez l'un et de l'absence chez l'autre d'un savoir préalable de la vérité des énoncés de foi (p. 83). Encore ici, donc, malgré leur accord théorique, ils s'engagent dans un désaccord que seule la conversion semble pouvoir surmonter (pp. 87-89).

À moins que le recours à la révélation permette de surmonter le désaccord? Penelhum consacre le chapitre suivant (c. 4) à l'analyse de ce concept. Devant les phénomènes révélateurs invoqués par le croyant, le sceptique a toujours le droit de rejeter l'interprétation théiste qui présuppose la connaissance de Dieu (p. 107). Mais ne peut-il pas y avoir des phénomènes révélateurs ayant une force probante, dont le refus serait irrationnel ? Faut-il exclure la possibilité de signes, c'est-à-dire de phénomènes connaissables, que l'on peut exprimer en termes non théistes, qui serviraient à révéler Dieu et à libérer l'affirmation théiste de tout doute raisonnable (p. 108) ? La réponse de Penelhum ne manque pas de perspicacité. Celui qui adopte le non-naturalisme théologique, écrit-il, qu'il soit croyant ou incroyant, n'attend pas de signes. Mais le croyant a-t-il raison de juger impossible la production d'un signe qui rendrait l'incroyance irrationnelle (p. 109) ? C'est ici, que Penelhum commence à douter de la sagesse du non-naturalisme théologique (p. 110). On ne peut pas en tout cas le justifier, souligne-t-il, en invoquant la liberté de la foi, c'est-à-dire en prétendant qu'un signe rendrait la foi nécessaire. 
Ce serait avoir une trop haute idée de la rationalité humaine. L'homme est toujours capable d'autodéception; il lui est toujours possible de connaître des prémisses, de savoir qu'elles sont vraies et qu'elles entraînent telle conclusion, et pourtant de refuser la vérité de cette dernière. En supprimant les raisons de douter, on ne force pas la foi. Devant un signe, le refus de l'incroyant est même libre, bien que déraisonnable et par conséquent blâmable. N'est-il d'ailleurs pas dans la logique du croyant d'estimer ainsi sans excuse la position de l'incroyant?

Il reste à Penelhum à tenter, dans un dernier chapitre, une interprétation de la nature de la foi (c. 5). Puisque c'est elle qui sépare le croyant de l'incroyant, c'est elle qu'il faut tenter de comprendre pour prendre conscience de leur position respective. L'effort de Penelhum vise surtout à corriger la façon habituelle de comprendre le rapport entre la foi et le savoir. Il importe, à son avis, en décrivant la foi, telle qu'elle est vécue, de retenir qu'elle est pour le croyant une sorte de savoir. Le philosophe ne peut pas dire qu'elle est vraiment un savoir ou n'en est pas un; s'il le faisait, il transgresserait la pure analyse et adopterait les critères de la foi ou ceux de l'incroyance (p. 132); mais il ne peut non plus décrire la foi sans dire qu'elle est vécue par le zroyant comme un savoir. Or, les principales théologies de la foi - celle de la foi - assentiment à un corps de propositions (saint Thomas), celle de la foi - interprétation globale de l'expérience (J. Hick) — se rendent incapables de décrire la foi comme savoir ; elles supposent inconciliables la dimension volontaire de la foi et la démonstration de ses énoncés. Thomas d'Aquin, par exemple, aurait soutenu que la foi est raisonnable dans la mesure où elle s'appuie à un savoir philosophique de Dieu, et qu'elle est volontaire dans la mesure où elle ne revêt pas ellemême la forme d'un savoir (p. 144). L'analyse de la logique de la foi tend au contraire à conclure, aux yeux de Penelhum, qu'elle n'a pas besoin d'être sans raison pour être libre, ni besoin de prémisses naturelles pour être raisonnable.

Dans ce reproche à saint Thomas d'avoir tantôt trop séparé la foi du savoir et tantôt d'avoir voulu appuyer la foi sur le savoir, se cache, me sembie-t-il, une erreur de perspective de la part de Penelhum. Le savorr auquel l'Aquinate oppose la foi n'est 
pas la même chose que le savoir dont, selon Penelhum, le croyant s'estime possesseur. Le knowledge de celui-ci a un sens différent de la scientia de celui-là. Il y a knowledge, si je comprends bien la conception courante des analystes, lorsque quelqu'un tient un énoncé pour vrai, en étant certain qu'il est vrai et en s'estimant justifié d'être certain de sa vérité. ${ }^{34}$ Or la foi de saint Thomas n'exclut jamais le savoir ainsi compris, étant faite de certitude et de cogitatio. Ce qu'elle exclut, c'est la saisie de la raison pour laquelle cet énoncé est nécessairement vrai et ne peut pas en être autrement, la démonstration au sens strict, propre à la scientia. ${ }^{35}$ Et je pense qu'il tenait là une position très sensée.

Nous n'avons pas affaire, bien sûr, à un travail d'historien. Mais puisque son auteur invite à contester le principe épistémologique dont se nourrit le désaccord entre le croyant et l'incroyant, le non-naturalisme théologique, puisque la théologie philosophique de Thomas d'Aquin représente une façon intelligente d'en sortir, il serait souhaitable de ne pas en rester à une image floue de ce que fut sa position sur le problème du rapport foi et raison.

Concluons par une question, celle-là même que les travaux de Penelhum forcent à poser: la neutralité dans l'analyse de la foi religieuse est-elle possible? Est-il vrai que la philosophie analytique fournit enfin un ontil logique, libre de toute idéologie, permettant d'analyser le discours religieux en mettant entre parenthèses la question de la vérité de la foi? Les travaux de Penelhum peuvent ne pas nous en convaincre, mais on aurait tort de méconnaître qu'ils offrent l'un des essais les plus intelligents et les plus honnêtes d'adoption jusqu'au bout de ce point de vue, les plus révélateurs aussi de ses limites.

\section{A. McKinnon et l'immunisation du langage de la foi contre la critique}

Le livre d'A. McKinnon, Falsification and Belief ${ }^{36}$ déploie une troisième possibilité de la philosophie analytique de la reli-

34. Ce sont les trois critères du knowledge proposés par A. J. AYER, The Problem of Knowledge, 1956, pp. 31-35.

35. Voir Summa Theologiae, II-IIe, q.1, a.5, ad $4 \mathrm{~m}$.

36. Alastair McKINNON, Falsiftcation and Belief, The Hague-Paris, Mouton, $1970,106 \mathrm{p}$. On trouvera une première rédaction de cet ouvrage dans "Unfalsifiability and the Uses of Religious Language», in American Pbilo- 
gion. Bien que publié avant les ouvrages de Nielsen et de Penelhum, il a pour objet de répondre à l'accusation portée par le premier de la non-falsifiabilité des énoncés de foi, de même qu’à la thèse, centrale chez le second, de leur indémonstrabilité. Par quelle stratégie? En analysant le langage religieux d'après son contexte propre, en refusant par conséquent d'en soumettre l'interprétation à une théorie préalable du sens, quitte à devoir contester certains dogmes établis de la tradition analytique. Exemple de fidéisme wittgensteinien, de balkanisation des jeux du langage pour immuniser le discours religieux contre la critique? Non. Dans son analyse de ce discours, McKinnon cherche au contraire à montrer que sa structure n'est pas unique, que le langage de la science, habituellement pris pour modèle d'analyse du sens, recèle la même logique, et que dans la science comme dans la foi il y a de l'infalsifiable et de l'indémontrable. Le résultat de cette stratégie, outre de critiquer la critique courante de la foi, sera de tendre la main à l'existentialisme.

L'analyse de McKinnon débute par l'examen d'un énoncé ( «Le monde a un ordre ) qui joue dans la pratique de la science un rôle analogue à celui des énoncés de foi ( «Dieu est amour», «Je crois en Dieu») dans la pratique religieuse. L'énoncé «le monde a un ordre » est employé par le savant de trois façons différentes et selon trois sens distincts. Il est d'abord employé comme énoncé asseriorique (assertional use), lorsque le savant veut exprimer un rapport causal observé ; dans ce cas, l'énoncé a un sens déterminé, renvoyant à un ordre particulier (p. 28). Le savant se sert du même énoncé, dans un autre contexte, par exemple lorsqu'il éprouve un échec dans sa recherche, non plus pour décrire le monde mais pour se rappeler la consigne impliquée dans son engagement à pratiquer la science : celle de traiter chaque événement comme s'il y avait un ordre dans le monde; dans ce contexte, l'emploi de l'énoncé est auto-instructif (selfinstructional), et son sens est purement heuristique (pp. 29-31).

sopbical Quarterly, 2 (1965), 229-237; «Unfalsifiability and Religious Belief 》, in Canadian Journal of Theology, 12 (1966), 118-125. Egalement du même auteur «Existence» in the «Existence of God», in American Philosophical Quarterly, 9 (1972), 349-352. Pour la discussion de la thèse de McKinnon, voir OGDEN, Schubert M., «Falsification and Belief», in Religious Studies, 10 (1974), 21-43. 
Enfin, dans un autre contexte plus rare, où le savant se voit forcé de justifier ses postulats, il fait un emploi méta-scientifique du même énoncé, emploi que McKinnon qualifie d'ontologico-linguistique (ontological-linguistic use) pour signifier que, dans ce cas, «le monde a un ordre» exprime l'intention de dire une vérité nécessaire (à la manière d'une convention linguistique) sur le monde (à la manière d'une affirmation d'existence) (pp. 32-39).

On voit les conséquences de cette analyse. Elle impose de rejeter comme reflétant mal la pratique du langage de la science le dogme hérité de Hume par la philosophie analytique, concernant l'impossibilité de jugements d'existence qui soient nécessairement vrais (pp. 43-44). Elle impose également de reconnaître qu'il y a de l'infalsifiable et de l'indémontrable dans la science: «le monde a de l'ordre » employé de façon auto-instructive ou ontologico-linguistique n'est aucunement falsifiable, et employé de façon ontologico-linguistique il est en outre tout à fait indémontrable (pp. 45-46), à peu près, ajouterions-nous, à la manière des premiers principes d'Aristote.

Après cette analyse, il ne reste plus à McKinnon qu'à montrer dans le discours religieux la mêtme diversité de sens (cc. 3 et 4). C'est souvent le tort du cririque, pense-t-il, de manquer de voir dans la foi l'exercice d'une pratique, la pratique, analogue à la science, d'une lecture du monde toujours orientée par des convictions préalables (p. 63). L'énoncé «Dieu est amour», par exemple, ne sert pas seulement à décrire Dieu à l'aide d'une représentation déterminée; il sert aussi à dire l'engagement à croire en l'amour de Dieu, et, lorsque le doute ou la critique l'impose, à exprimer ce que le fidèle tient pour justification dernière de sa foi.

Arrêtons-nous un moment à cette troisième manière ontologico-linguistique - d'employer l'énoncé de foi. Il exprime alors pour le croyant une vérité évidente et nécessaire, soustraite à toute démonstration, immunisée par conséquent contre toute critique (pp. 81-88). Qu'exprime-t-il au juste? Un concept purement heuristique indéterminé, de Dieu. Rien d'autre, pense McKinnon, ni l'affirmation d'un fait, ni la conclusion des preuves de l'existence de Dieu, ne peut assurer à la pratique de la foi sa rationalité et sa cohérence. Il faut à tout prix qu'au principe de la 
foi se trouve énoncée une sorte de conscience, immédiate (insight) - que McKinnon n'ose pas appeler foi - de la transcendance de Dieu par rapport à toute représentation (p. 83). La représentation joue dans la foi un rôle indispensable, mais secondaire et dérivé par rapport à ce sens heuristique de Dieu, sorte de régulateur interne permettant au croyant de corriger l'inévitable déficience de ses représentations et d'en faire l'index de Dieu (p. 98).

On n'a pas de peine à voir dans cette thèse de McKinnon la présence de deux points de vue différents : celui de l'analyse du langage, de style post-Wittgensteinien, et celui de la théologie existentialiste de Kierkegaard à Tillich. Le premier point de vue lui permet de décrire le langage de la foi, de montrer au critique qu'il ne le comprend pas, que sur lui repose le fardeau de prouver que le «Dieu » du fidèle manque d'intelligibilité ou de cohérence (p. 103). Le second point de vue lui permet de comprendre la foi comme une pratique herméneutique, une interprétation originale du monde, orientée par une idée, qui justifie cette pratique et se trouve justifiée par elle.

Cette thèse ne manque pas de grandeur. Elle n'est pas sans évoquer, par delà la théologie existentialiste, l'apophatisme de la tradition néo-platinicienne. ${ }^{37}$ Elle demeure toutefois une thèse qui impose au sceptique et au croyant des demandes trop onéreuses pour résoudre à leur satisfaction le problème du rapport entre la raison et la foi. Au sceptique, elle demande non seulement d'admettre qu'il ne comprend rien au langage de la foi, mais de se résoudre à tenir pour irréparable la rupture de communication entre la foi et l'incroyance. Du croyant, elle réclame une attitude ambiguë : voir dans l'énoncé de foi une vérité évidente et nécessaire, tout en sachant que sa vérité est seulement hypothétique, ${ }^{38}$ confesser par conséquent qu'il a pu se tromper en décidant de croire, mais qu'une fois choisie la pratique de la foi, il doit se comporter comme si elle était indiscutablement vraie.

37. Comme il se trouve exposé par exemple, dans l'ouvre d'H. Dumery, beaucoup plus conscient que McKinnon de ses postulats.

38. "There is no absolute and unconditional justification of belief as such... The justification for belief is like that of science; in both cases it is finally conditioned. 》 (p. 104). 


\section{King-Farlow et Christensen ou le refus d'immuniser la foi contre la critique}

Il me reste, avant de conclure, à dire un mot de l'ouvrage de J. King-Farlow et W. N. Christensen, Faith and the Life of Reason. ${ }^{39}$ Il s'agit d'un recueil d'articles, retravaillés bien sûr, réédités, dont je déplore qu'on ne nous donne sur leur première publication aucun renseignement précis. En outre, le lien entre les huit chapitres de ce livte est trop artificiel pour offrir sur les problèmes discutés par la philosophie analytique de la religion une thèse bien articulée. Enfin, c'est peut-être faire injure à KingFarlow et Christensen que d'insérer leur ouvrage dans ce bulletin sur la philosophie analytique de la religion, car leurs liens avec cette tradition, dont ils critiquent la passion antimétaphysique et les dogmes fondamentaux, sont assez lâches, et la position qu'ils défendent se réclame davantage de Thomas d'Aquin et de William James que de Wittgenstein.

La raison pour laquelle je tiens à clore ce bulletin par la discussion de ce livre, c'est qu'il traduit une nouvelle préoccupation dans la philosophie anglo-américaine de la religion : celle de tirer au clair à la fois les possibilités et les problèmes proposés à la foi religieuse par le rationalisme critique de $\mathrm{K}$. Popper. Il semble que la philosophie de la religion soit toujours un peu en retard sur les autres disciplines philosophiques et qu'elle prend toujours plus de temps à s'éveiller aux problèmes soulevés par une nouvelle épistémologie. Ajoutons à cela que de ce côté-ci de l'Atlantique on met du temps à liquider les vieux débats qui ont occupé jadis la scène philosophique en Angleterre et nous com. prendrons pourquoi l'on ne fait que commencer, en philosophie de la religion, à réfléchir la critique de la foi, inspirée par l'épistémologie popperienne, ${ }^{40}$ qui ne vise plus le sens du langage religieux, mais la foi elle-même, accusée de trahir la raison au profit d'une irrationnelle « retreat to commitment».

39. John KING-FARLOW and William Niels CHRISTENSEN, Faith and the Life of Reason, Dordrecht, Reider, 1972, xiii, 253 p.

40. Je dis bien «inspirée» par l'épistémologie de Popper. On ne trouve chez ce dernier aucune critique de la religion. C'est cependant de son modèle de rationalité que s'inspire la critique de W. W. BARTLEY, The Retreat to Commitment, London, 1962, de même que celle, beaucoup moins sereine, développée dans les écrits de Hans ALBERT. Voir note suivante. 
Comme réponse du croyant à cette accusation, King-Farlow et Christensen proposent une «méthodologie empiriste et libérale » (p. 3), consistant à adopter à l'égard des énoncés théologiques une attitude semblable à celle du savant devant les hypothèses scientifiques, c'est-à-dire une attitude d'essai d'explication de l'expérience (p. 2). Essayons de comprendre ce qu'implique, pour nos auteurs, l'adoption de cette attitude par le croyant. Elle implique d'abord, soulignent-ils, un engagement préalable au choix d'une idéologie religieuse : l'engagement à chercher le vrai, à faire le bien, à réaliser la justice (p. 4). Seul cet engagement rend possibles le respect mutuel et la coopération réciproque des tenants d'idéologies opposées. Dieu veut que nos engagements idéologiques jaillissent d'un engagement plus fondamental à l'égard du vrai et du bien; telle est, selon nos auteurs, la formule du théisme antivolontariste. Le volontariste, adversaire par excellence du croyant libéral de tempérament empiriste, pense au contraire «qu'il est sage de croire parce que Dieu le veut», et cette proposition chez lui exclut ou précède cette autre «Dieu veut que nous croyions et nous devrions croire parce que comme le montre la réflexion critique - cela est sage et juste » (p. 4).

L'adoption de cette méthodologie implique, en deuxième lieu, que le critère du croyant pour juger satisfaisante une idéologie religieuse sera avant tout son aptitude à fournir une explication justificatrice de l'existence du monde et de notre expérience du monde (p. 5). Par explication justificatrice nos auteurs entendent plus qu'une explication causale ; il s'agit d'une explication faisant appel à une cause ultime qui rend compte du fait qu'il y a du sens et de la valeur dans tout ce qui arrive (ibid).

Ce n'est pas tout, ce n'est même pas encore le plus important. L'adoption par le croyant de cette méthodologie implique surtout qu'il renonce à soustraire le contenu de sa foi à la critique, et qu'il consente à le tenir pour falsifiable. Tenir l'énoncé «Dieu existe» pour non falsifiable, écrivent nos auteurs, c'est ne pas être fondamentalement engagé pour la vérité, ni même pour la rationalité (p. 7). On voit que ce que recommandent nos auteurs, c'est plus qu'une attitude, rien de moins qu'un modèle de rationalité, bien connu des lecteurs de Popper et de Quine, 
adapté ici au programme d'un théisme pragmatique. D'après ce modèle, ce qui fait la rationalité d'une doctrine ce n'est pas tant son contenu que la manière d'y adhérer (p. 8) : avec détachement, sans dogmatisme, n'immunisant rien contre la critique, ${ }^{41}$ consentant à la voir réfutée et à admettre que l'on s'est trompé, tout comme le savant qui procède par «conjectures et réfutations».

Du reste du livre de King-Farlow et Christensen, je dirai peu de choses. Je soulignerai seulement leur effort, louable à mon avis, pour réhabiliter, contre la majorité des analystes, la notion d'Être nécessaire et pour ouvrir la raison à des propositions d'existence qui sont nécessaires (pp. 78-154). Quant à leur traitement des attributs de Dieu, il m'a paru refléter trop de bonhomie, touchant parfois à la légèreté. Soutenir en tout cas que le concept d'un dieu immuable et intemporel chez Thomas d'Aquin lui est venu de son attachement à Áristote et n'a rien à faire avec le message biblique (pp. 112 ss) me paraît traduire un manquè évident de sens de l'histoire. La théologie augustinienne, par exemple, que personne ne saurait soupçonner de lien avec l'aristotélisme, accordait pourtant à l'immutabilité de Dieu le rôle de premier des attributs de Dieu. Pour ce qui est du choix proposé aux croyants entre l'empirisme logique de Nielsen, Carnap, Ayer et le théisme pragmatique de nos auteurs, entre la thérapie linguistique et Primaxeput (Principle of Maximizing Expected Utility) (p. 221), je laisse au fidèle le soin de ne pas s'y laisser prendre.

Il reste que la stratégie pratiquée par King-Farlow et Christensen, de même que le modèle de rationalité qui l'inspire, soulèvent une question capitale. Est-il légitime de faire jouer aux énoncés de foi le rôle d'bypothèses dans la vie du croyant, plutôt

41. L'expression «tactique ou stratagème d'immunisation d'un thème contre la critique» fut d'abord employée par Hans Albert et connut beaucoup de succès. Karl Popper l'a intégrée avec beaucoup de reconnaissance dans son vocabulaire. Voir K. Popper, Replies to my Critics, in The Pbilosopby of Karl Popper, ed. by P. A. Schilpp, Library of Living Philosophers, La Salle, Ill., 1974, Vol. 2, p. 983. J'avoue pour ma part trouver étrange que la méthodologie popperienne, d'esprit si antidogmatique, serve à Hans Albert à défendre avec passion le dogme de l'irrationalité de la foi. Voir ses deux principaux ouvrages: Traktat über kritische Vermunft, Tübingen, Mohr, 1969, 190 p. ; Plädoyer für kritischen Rationalismus, München, Piper, 1971, $149 \mathrm{p}$. 
que le rôle de principes, comme la certitude propre à la foi religieuse semblerait le suggérer? Je pense qu'il faudrait, pour retenir ce qu'il y a de vrai dans la thèse de nos auteurs, sans toutefois mutiler la certitude vécue du croyant, bien distinguer entre la fonction de la foi dans la vie humaine et son fondement. Sa fonction ressemble étroitement à celle d'une hypothèse scientifique, et pour cela le procédé de King-Farlow et Christensen paraît jusqu'à un certain point légitime. La foi a pour rôle, en effet, de rendre compte de l'expérience dans sa totalité. Elle s'adresse à une raison de vivre qui ne s'impose jamais avec l'évidence d'un principe ou d'une conclusion démontrée. Cette raison de vivre, le croyant doit constamment la soumettre à l'épreuve de l'expérience, avec le réalisme de celui qui sait que le réel triomphe toujours de nos illusions. Jusque là je n'éprouve aucune gêne à faire route avec nos deux auteurs. S'ils demandent cependant de penser que la foi, comme l'hypothèse pour le savant, a pour seul fondement l'ensemble des faits à expliquer, et que, pour cela, le croyant doit la tenir pour réfutable, je suis obligé de prendre une autre route, sous peine de passer pour un volontariste irrécupérable.

$$
\text { **** } * *
$$

Les huit ouvrages analysés dans ce bulletin sont assez représentatifs de ce qui se fait en philosophie analytique de la religion pour faire voir l'ambiguiité de cette discipline. Ce qui étonne dans ce genre d'analyse de la religion, c'est avant tout le fait qu'elle contribue si peu à comprendre le christianisme, la seule religion habituellement étudiée par les analystes. ${ }^{42}$ Comparée à l'exégèse, l'histoire, voire la théologie, elle fait pauvre figure. On dira qu'elle n'est pas une science de la religion, qu'elle n'a pas pour but de comprendre le christianisme, mais seulement de filtrer ce qu'il contient de raisonnable. Mais le philosophe de la religion ne se propose-t-il pas de faire à l'égard de la religion ce que l'épistémologue fait à l'égard de la science, c'est-à-dire articuler sa logique et, par là, contribuer à une pratique plus intelligente

42. Il n'entrait pas dans les limites de ce bulletin de présenter les travaux du prof. E. L. FACKENHEIM de Toronto. Je me permets seulement de faire remarquer que sa récente étude, God's Presence in History: Jewish Affirm. ations and Pbilosopbical Reflections, N.Y., Harper, 1970, 104 p., contribue incomparablement mieux à faire comprendre la foi d'Israël que ne le font pour le christianisme beaucoup de travaux des analystes. 
de la science? Et pourtant, qui n'admettra que si l'on apprend beaucoup sur la nature de la science à lire les épistémologues, on apprend très peu sur la religion à fréquenter ses analystes.

On comprend mal, en réalité, l'intentionnalité de la philosophie de la religion si l'on attend d'elle qu'elle contribue à une meilleure intelligence de la religion. Ce n'a jamais été son souci de réfléchir la pratique de la foi ; ce qu'elle réfléchit, ce sont les exigences de la raison devant la religion. C'est dans le but de préserver l'autonomie et la liberté de la raison qu'elle a pris forme, entre la théologie et les sciences de la religion, et c'est le même but qui l'inspire encore. Lire un ouvrage de philosophie de la religion, qu'il soit de style analytique ou autre, ce n'est probablement pas la meilleure façon de se renseigner sur l'esprit d'une religion, mais c'est un excellent moyen de prendre conscience des modèles de rátionalité les plus pertinents.

Que dire du modèle de rationalité dont se nourrit la philosophie analytique de la religion? Il m'a semblé manquer souvent de souplesse. Il exprime assez bien ce que fait la raison dans la pratique de certaines sciences, ou dans une certaine pratique de la science, celle où la raison ne se laisse mesurer par tien d'autre qu'elle-même; mais rendre compte des démarches étrangères à la pratique qu'il symbolise, il en est incapable. La critériologie qu'il propose dit bien clairement ce qu'il faut tenir pour contraire à la raison; quant à dicter ce qu'il faut tenir pour vrai, elle en paraît impuissante.

Université d'Ottawa 